\title{
A MODIFIED CONVOLUTION AND PRODUCT THEOREM FOR THE LINEAR CANONICAL TRANSFORM DERIVED BY REPRESENTATION TRANSFORMATION IN QUANTUM MECHANICS
}

\author{
NAVDEEP GOEL* , KULBIR SINGH** \\ * Electronics and Communication Engineering Section, Yadavindra College of Engineering \\ Punjabi University Guru Kashi Campus, Talwandi Sabo-151302, Punjab, India \\ e-mail: navdeepgoel.ycoe@gmail.com \\ ** Department of Electronics and Communication Engineering \\ Thapar University, Patiala-147001, Punjab, India
}

\begin{abstract}
The Linear Canonical Transform (LCT) is a four parameter class of integral transform which plays an important role in many fields of signal processing. Well-known transforms such as the Fourier Transform (FT), the FRactional Fourier Transform (FRFT), and the FreSnel Transform (FST) can be seen as special cases of the linear canonical transform. Many properties of the LCT are currently known but the extension of FRFTs and FTs still needs more attention. This paper presents a modified convolution and product theorem in the LCT domain derived by a representation transformation in quantum mechanics, which seems a convenient and concise method. It is compared with the existing convolution theorem for the LCT and is found to be a better and befitting proposition. Further, an application of filtering is presented by using the derived results.
\end{abstract}

Keywords: linear canonical transform, convolution and product theorem, quantum mechanical representation.

\section{Introduction}

The Linear Canonical Transform (LCT) (Alieva and Bastiaans, 1999; Moshinsky and Quesne, 1971; Nazarathy and Shamir, 1982; Pei and Ding, 2001; 2002; Hennelly and Sheridan, 2005) is a four parameter $(a, b, c, d)$ class of linear integral transform. It was introduced in the 1970s (Moshinsky and Quesne, 1971) and has found many applications in signal processing, radar system analysis, filter design, phase retrieval, pattern recognition (Shin and Park, 2011; Krzysztof and Domańska, 2011), signal synthesis, and many other areas (Stern, 2006; Tao et al., 2004; Ozaktas et al., 2000; Almeida, 1994; Barshan et al., 1997; Sharma and Joshi, 2006; Goel and Singh, 2011). Well-known transforms such as the Fourier Transform (FT), the FRactional Fourier Transform (FRFT), and the FreSnel Transform (FST) can be seen as special cases of the linear canonical transform (Tao et al., 2004; Ozaktas et al., 2000; Almeida, 1994). Origins of LCTs are in quantum mechanics; a brief overview may be found in the work of Wolf (1979). As a generalization of the FT and FRFT, the basic theories of the LCT have been developed including sampling the signals (Deng et al., 2006; Li et al., 2007; Koc et al., 2008; Healy and Sheridan, 2008; Tao et al., 2008), discrete approximations to the transforms (Pei and Ding, 2002; Hennelly and Sheridan, 2005; Healy and Sheridan, 2009; Oktem and Ozaktas, 2010), and so on, which can enrich the theoretical system of the LCT.

Many properties of the LCT and FRFT are already known (Alieva and Bastiaans, 1999; Pei and Ding, 2002; Ozaktas et al., 2000), including the product and convolution theorem (Deng et al., 2006; Ozaktas et al., 1994; Almeida,1997; Zayed,1998; Sharma and Joshi, 2007; Wei et al., 2012; 2009; Singh and Saxena, 2011), but none have received acclamation because either their definitions do not generalize very nicely to the classical definition for the FT or result in a bigger hardware complexity due to a large number of chirp signals.

In mathematics and, in particular, functional analysis, convolution is a mathematical operation on two functions $f$ and $g$, producing a third function that is typically viewed as a modified version of one of the original functions, giving the area overlap between the 
two functions as a function of the amount by which one of the original functions is translated. In other words, the Fourier transform of the convolution of two signals is the point-wise product of the Fourier transform of their respective signals. In the literature, many definitions of the convolution theorem for the LCT (Deng et al., 2006; Wei et al., 2012; 2009) are proposed. The classical definition of the convolution and product theorem of the FT for the signals $f(x)$ and $g(x)$ is given by

Convolution:

$$
\begin{aligned}
f(x) & \otimes g(x) \\
= & \int_{-\infty}^{\infty} f(y) g(x-y) \mathrm{d} y \stackrel{F T}{\longleftrightarrow} \sqrt{2 \pi} F(p) G(p),
\end{aligned}
$$

Product:

$$
f(x) \cdot g(x) \stackrel{F T}{\longleftrightarrow} F(p) \otimes G(p),
$$

where $F(p)$ and $G(p)$ are the FTs of $f(x)$ and $g(x)$, respectively, and ' $\otimes$ ' denotes the convolution operation.

In this paper, a modified convolution and product theorem is proposed with a new proof from the quantum mechanical representations transform, which seems simple and elegant. With the help of simulations, an attempt is made to compare the simulation results of the proposed theorem with the theorems proposed in the literature and it is found that the proposed theorem is a better proposition and can be considered a generalization of the classical convolution theorem of the FT. In the application section, a filter is designed by using the derived results.

\section{Brief review of the correspondence between the LCT and its quantum mechanical counterpart}

The LCT is also known as the generalized Fresnel transform (James and Agarwal,1996; Palma and Bagini, 1997), the ABCD transform (Bernardo, 1996), the Collins formula (Collins, 1970), generalized Huygens integrals (Nazarathy and Shamir, 1982), quadratic-phase integrals or quadratic-phase systems (Bastiaans, 1979), extended fractional Fourier transforms (Hua et al., 1997) as well as Moshinsky and Queesne integrals (Moshinsky and Quesne, 1971), and is a special case of the special affine Fourier transform (Abe and Sheridan, 1994a; 1994b). The one-dimensional LCT with parameters of a signal $f(x)$ is defined (Alieva and Bastiaans, 1999) as

$$
\begin{aligned}
& L_{(a, b, c, d)}[f(x)](p) \\
& \quad= \begin{cases}\int_{-\infty}^{+\infty} f(x) K_{(a, b, c, d)}(p, x) \mathrm{d} x, & b \neq 0, \\
\sqrt{d} e^{j(c d / 2) p^{2}} f(d \cdot p), & b=0,\end{cases}
\end{aligned}
$$

where parameters $(a, b, c, d)$ are real numbers and the matrix $\left(\begin{array}{ll}a & b \\ c & d\end{array}\right)$ belongs to $\operatorname{SL}(2, \mathbb{R})$.

In this paper, we only consider the case of $b \neq 0$ since the LCT is just a chirp multiplication operation if $b=0$. The term $K_{(a, b, c, d)}(p, x)$ represents the integral kernel and is given by

$$
\begin{aligned}
& K_{(a, b, c, d)}(p, x) \\
&=\sqrt{\frac{1}{j 2 \pi b}} \exp \left[\frac{j\left(a x^{2}+d p^{2}\right)}{2 b}-\frac{j x p}{b}\right] .
\end{aligned}
$$

As special cases of the LCT, when $(a, b, c, d)=$ $(\cos \alpha, \sin \alpha,-\sin \alpha, \cos \alpha)$, the LCT reduces to the FRFT, and when $(a, b, c, d)=(0,1,-1,0)$ the LCT reduces to the FT.

To relate the LCT kernel in a quantum mechanical representation, we use the completeness relation of the coherent state with the arguments of $z_{1}$ and $z_{2}$. The coherent state $|z\rangle$ is defined (Zhang et al., 1990) by the eigenstate of the annihilation operator $a$ with the complex eigenvalue $z$, i.e.,

$$
a|z\rangle=z|z\rangle
$$

and the completeness relation (Ogura and Sekiguchi, 2007)

$$
\int \frac{\mathrm{d}^{2} z}{2 \pi j}|z\rangle\langle z|=1 .
$$

To relate the LCT kernel in quantum mechanics, we use the completeness relation of the coherent state with the arguments of $z_{1}$ and $z_{2}$,

$$
\begin{aligned}
& \langle Q|U(t)| q\rangle \\
& \quad=\int \frac{\mathrm{d}^{2} z_{1} \mathrm{~d}^{2} z_{2}}{(2 \pi j)^{2}}\left\langle Q \mid z_{1}\right\rangle\left\langle z_{1}|U(t)| z_{2}\right\rangle\left\langle z_{2} \mid q\right\rangle
\end{aligned}
$$

where

$$
\int \mathrm{d}^{2} z \equiv \int \mathrm{d}[\operatorname{Re}(z)] \mathrm{d}[\operatorname{Im}(z)],
$$

and with the aid of the Integration Within Ordered Product (IWOP) technique (Fan and VanderLinde, 1989; Fan et al., 1987) the coherent state representation of the unitary operator is given by

$$
\begin{aligned}
\left\langle z_{1}|U(t)| z_{2}\right\rangle= & \frac{1}{\sqrt{s}} \exp \left[-\frac{r}{2 s}\left(z_{1}^{*}\right)^{2}+\frac{z_{2} z_{1}^{*}}{s}+\frac{r^{*}}{2 s}\left(z_{2}\right)^{2}\right. \\
& \left.-\frac{\left|z_{1}\right|^{2}}{2}-\frac{\left|z_{2}\right|^{2}}{2}\right],
\end{aligned}
$$

where $(r, s),\left|s^{2}\right|-\left|r^{2}\right|=1$, are related to a classical ray transfer matrix $\left(\begin{array}{ll}a & b \\ c & d\end{array}\right)$ by

$$
s=\frac{1}{2}[a+d+j(b-c)], \quad r=\frac{1}{2}[d-a-j(b+c)],
$$


the uni-modularity condition $a d-b c=1$ is equivalent to $\left|s^{2}\right|-\left|r^{2}\right|=1$, and the coordinate and momentum representation of the coherent state $|z\rangle$ (Ogura, 2009)

$$
\begin{aligned}
& \left\langle Q \mid z_{1}\right\rangle=\frac{1}{\pi^{1 / 4}} \exp \left[-\frac{Q^{2}}{2}+\sqrt{2} z_{1} Q-\frac{z_{1}^{2}}{2}-\frac{\left|z_{1}\right|^{2}}{2}\right]_{(8}, \\
& \left\langle q \mid z_{2}\right\rangle=\frac{1}{\pi^{1 / 4}} \exp \left[-\frac{q^{2}}{2}-j \sqrt{2} z_{2} q+\frac{z_{2}^{2}}{2}-\frac{\left|z_{2}\right|^{2}}{2}\right] .
\end{aligned}
$$

By substituting the values of Eqns. (6), (8) and (9) in Eqn. (5), we get

$$
\begin{aligned}
& \langle Q|U(t)| q\rangle \\
& =\int \frac{\mathrm{d}^{2} z_{1} \mathrm{~d}^{2} z_{2}}{(2 \pi j)^{2}} \times \frac{1}{\pi^{1 / 2}} \frac{1}{\sqrt{s}} \\
& \cdot \exp \left[-\frac{Q^{2}}{2}+\sqrt{2} z_{1} Q-\frac{z_{1}^{2}}{2}-\frac{\left|z_{1}\right|^{2}}{2}\right] \\
& \cdot \exp \left[-\frac{r}{2 s}\left(z_{1}^{*}\right)^{2}+\frac{z_{2} z_{1}^{*}}{s}+\frac{r^{*}}{2 s}\left(z_{2}\right)^{2}-\frac{\left|z_{1}\right|^{2}}{2}-\frac{\left|z_{2}\right|^{2}}{2}\right] \\
& \cdot \exp \left[-\frac{q^{2}}{2}+\sqrt{2} z_{2}^{*} q-\frac{\left(z_{2}^{*}\right)^{2}}{2}-\frac{\left|z_{2}\right|^{2}}{2}\right] .
\end{aligned}
$$

This results from solving for $z_{1}$ and using the fact (Puri, 2001) that, if $a_{1}^{2}-4\left|c_{1}\right|^{2}>0$, then

$$
\begin{aligned}
& \frac{1}{\pi} \int \mathrm{d}^{2} \alpha \exp \left(-a_{1}|\alpha|^{2}+b_{1} \alpha+b_{1}^{*} \alpha^{*}+c_{1} \alpha^{2}+c_{1}^{*}\left(\alpha^{*}\right)^{2}\right) \\
& =\sqrt{\frac{1}{a_{1}^{2}-4\left|c_{1}\right|^{2}}} \exp \left[\frac{b_{1}^{2} c_{1}^{*}+b^{* 2} c_{1}+a_{1}\left|b_{1}\right|^{2}}{a_{1}^{2}-4\left|c_{1}\right|^{2}}\right]
\end{aligned}
$$

and

$$
\begin{aligned}
& \frac{1}{\pi} \int \mathrm{d}^{2} z_{1} \exp \left[-\left|z_{1}\right|^{2}+z_{1} \sqrt{2} Q+\frac{z_{2} z_{1}^{*}}{s}-\frac{z_{1}^{2}}{2}-\frac{r}{2 s}\left(z_{1}^{*}\right)^{2}\right] \\
& =\sqrt{\frac{s}{s-r}} \exp \left[\frac{-Q^{2} r-\frac{z_{2}^{2}}{2 s}+\sqrt{2} Q z_{2}}{s-r}\right] .
\end{aligned}
$$

Here $a_{1}=1, b_{1}=\sqrt{2} Q, b_{1}^{*}=z_{2} / s, c_{1}=-1 / 2$, and $c_{1}^{*}=-r / 2 s$. Similarly, solving for $z_{2}$ and substituting the value in Eqn. (10), we finally get

$\langle Q|U(t)| q\rangle$

$$
\begin{aligned}
& =\sqrt{\frac{1}{\pi\left(s-s^{*}-r+r^{*}\right)}} \exp \left[\frac{2 q Q}{s-s^{*}-r+r^{*}}\right. \\
& \left.-\frac{q^{2}}{2} \frac{s+s^{*}-r-r^{*}}{s-s^{*}-r+r^{*}}-\frac{Q^{2}}{2} \frac{s+s^{*}+r+r^{*}}{s-s^{*}-r+r^{*}}\right] .
\end{aligned}
$$

By substituting the value of $r$ and $s$ from Eqn. (7), the LCT kernel is given by

$$
\langle Q|U(t)| q\rangle=\sqrt{\frac{1}{j 2 \pi b}} \exp \left[\frac{j}{2 b}\left(a q^{2}-2 q Q+d Q^{2}\right)\right] \text {. }
$$

As a special case of the LCT, when $(a, b, c, d)=$ $(\cos \alpha, \sin \alpha,-\sin \alpha, \cos \alpha)$, Eqn. (13) reduces to the kernel of the fractional Fourier transform (Namias, 1979) $K_{\alpha}(Q, q)$ as

$K_{\alpha}(Q, q)=\sqrt{\frac{1}{2 j \pi \sin \alpha}} \exp \left[\frac{j}{2}\left(\frac{q^{2}+Q^{2}}{\cot \alpha}-\frac{2 q Q}{\sin \alpha}\right)\right]$.

Multiplying and dividing Eqn. (13) by $\sqrt{\exp (j \alpha)}$, we finally get

$L_{(\cos \alpha, \sin \alpha,-\sin \alpha, \cos \alpha)}[x(q)]=\sqrt{\exp (-j \alpha)} F_{\alpha}[x(q)]$,

where $F_{\alpha}[x(q)]$ denotes the fractional Fourier transform of $x(q)$. As a special case of the LCT, when $(a, b, c, d)=$ $(0,1,-1,0)$, Eqn. (13) reduces to the kernel of Fourier transform as

$$
L_{(0,1,-1,0)}[x(q)]=\sqrt{-j} F[x(q)],
$$

where $F[x(q)]$ denotes the Fourier transform of $x(q)$. Hence the LCT can extend its utilities and applications and solve some problems that cannot be solved well by the operations defined by Ozaktas et al. (2000).

\section{Modified identities for the convolution and product theorem}

The new definition of the convolution and product theorem for the LCT is as follows.

Theorem 1. (Convolution) If $F_{(a, b, c, d)}(p)$ is the LCT of $f(x)$ and $G_{(a, b, c, d)}(p)$ is the LCT of $g(x)$, then

$$
\sqrt{j 2 \pi b} e^{-\frac{j}{2}\left(\frac{d}{b} p^{2}\right)} F_{(a, b, c, d)}(p) G_{(a, b, c, d)}(p)
$$

is a linear canonical transform of $h(x)$, i.e.,

$$
\begin{aligned}
& L_{(a, b, c, d)}(h(x)) \\
& =\sqrt{j 2 \pi b} \exp \left[-\frac{j}{2}\left(\frac{d}{b} p^{2}\right)\right] \\
& \quad \times F_{(a, b, c, d)}(p) G_{(a, b, c, d)}(p),
\end{aligned}
$$

where

$$
h(x)=\int_{-\infty}^{\infty} f(y) g(x-y) \tilde{y}(x, y) \mathrm{d} y,
$$

is the weighted convolution and the weight function is $\tilde{y}(x, y)=e^{-j \frac{a}{b} y(x-y)}$ while the role of $g$ and $f$ can be interchanged. 
Theorem 2. (Product) For any two functions $f(x)$ and $g(x)$, the modified product operation is defined as

$$
\begin{gathered}
m(x)=g(x) \cdot f(x) \cdot e^{j \frac{a}{2 b} x^{2}} \\
L_{(a, b, c, d)}\left(g(x) \cdot f(x) \cdot e^{j \frac{a}{2 b} x^{2}}\right) \\
=G_{(a, b, c, d)}(p) \otimes F_{(a, b, c, d)}(p) \cdot \exp \left[j \frac{d}{b} v p\right] .
\end{gathered}
$$

\section{Proposed transform of a convolution by coordinate-momentum representation transform}

Using the quantum mechanical notation (Fan and Yue, 2003; Fan et al., 2008), the one-dimensional linear canonical transform of the signal $h(x)$ reads as

$$
\begin{aligned}
& L_{(a, b, c, d)}(h(x)) \\
& \quad=\frac{1}{\sqrt{j 2 \pi b}} \int_{-\infty}^{\infty} \mathrm{d} x\langle p|K| x\rangle\langle x \mid h\rangle=\langle p|K| h\rangle \\
& \quad=\langle p \mid H\rangle=H_{(a, b, c, d)}(p),
\end{aligned}
$$

where we use the notation $\langle x \mid h\rangle=h(x)$ and $\langle p|K| x\rangle$ gives the representation of the LCT kernel in quantum mechanics. $K$ is named as the LCT operator and

$$
\langle x \mid h\rangle=\int_{-\infty}^{\infty} \mathrm{d} y\langle y \mid f\rangle\langle x-y \mid g\rangle \exp \left[-j \frac{a}{b} y(x-y)\right] .
$$

Substituting the value of Eqn. (18) in (17) results in

$$
\begin{aligned}
L_{(a, b, c, d)}(h(x)) & \\
= & \frac{1}{\sqrt{j 2 \pi b}} \int_{-\infty}^{\infty} \mathrm{d} x\langle p|K| x\rangle \int_{-\infty}^{\infty} \mathrm{d} y\langle y \mid f\rangle\langle x-y \mid g\rangle \\
& \cdot \exp \left[-j \frac{a}{b} y(x-y)\right]
\end{aligned}
$$

Rearranging Eqn. (19) we get

$$
\begin{aligned}
& L_{(a, b, c, d)}(h(x)) \\
& =\frac{1}{\sqrt{j 2 \pi b}} \int_{-\infty}^{\infty} \int_{-\infty}^{\infty} \mathrm{d} x \mathrm{~d} y\langle p|K| x\rangle\langle x-y \mid g\rangle\langle y \mid f\rangle \\
& \quad \cdot \exp \left[-j \frac{a}{b} y(x-y)\right]
\end{aligned}
$$

Substituting $x-y=x^{\prime}$, i.e., $x=x^{\prime}+y$ and $y=y$, in Eqn. (20), we have $\mathrm{d} x \mathrm{~d} y=\mathrm{d} x^{\prime} \mathrm{d} y$ (Kiusalaas, 2010), and then replacing $x^{\prime}$ by $x$ results in

$$
\begin{aligned}
& L_{(a, b, c, d)}(h(x)) \\
& =\frac{1}{\sqrt{j 2 \pi b}} \int_{-\infty}^{\infty} \int_{-\infty}^{\infty} \mathrm{d} x \mathrm{~d} y\langle p|K| x+y\rangle\langle x \mid g\rangle\langle y \mid f\rangle \\
& \quad \cdot \exp \left[-j \frac{a}{b} y x\right] .
\end{aligned}
$$

Rewriting $\langle p|K| x+y\rangle$ explicitly, we get

$$
\begin{aligned}
& \langle p|K| x+y\rangle \frac{1}{\sqrt{j 2 \pi b}} \exp \left[-j \frac{a}{b} y x\right] \\
= & \frac{1}{\sqrt{j 2 \pi b}} \\
& \quad \cdot \exp \left[\frac{j d p^{2}-2 j p(x+y)+j a(x+y)^{2}-2 j a x y}{2 b}\right] .
\end{aligned}
$$

Multiplying and dividing Eqn. (22) by $\exp \left[\frac{j}{2} \frac{d}{b} p^{2}\right] / \sqrt{j 2 \pi b}$ result in

$$
\begin{aligned}
\Rightarrow & \frac{\sqrt{j 2 \pi b}}{j 2 \pi b} \\
& \cdot \exp \left[\frac{j d p^{2}-2 j p x-2 j p y+j a x^{2}+j a y^{2}}{2 b}\right] \\
& \cdot \exp \frac{j d p^{2}-j d p^{2}}{2 b}
\end{aligned}
$$

Rearranging Eqn. (23) we get

$$
\begin{aligned}
& \Rightarrow \frac{\sqrt{j 2 \pi b}}{j 2 \pi b}\left[\exp \left\{\frac{j d p^{2}-2 j p x+j a x^{2}}{2 b}\right\}\right. \\
& \left.\cdot \exp \left\{\frac{j d p^{2}-2 j p y+j a y^{2}}{2 b}\right\} \exp \left\{\frac{-j d p^{2}}{2 b}\right\}\right] .
\end{aligned}
$$

Substituting Eqn. (24) in (21) results in

$$
\begin{aligned}
L_{(a, b, c, d)}(h(x)) & \frac{\sqrt{j 2 \pi b}}{j 2 \pi b} \int_{-\infty}^{\infty} \int_{-\infty}^{\infty} \mathrm{d} x \mathrm{~d} y\langle x \mid g\rangle\langle y \mid f\rangle \\
& \cdot\left[\exp \left\{\frac{j d p^{2}-2 j p x+j a x^{2}}{2 b}\right\}\right. \\
& \left.\cdot \exp \left\{\frac{j d p^{2}-2 j p y+j a y^{2}}{2 b}\right\} \cdot \exp \left\{\frac{-j d p^{2}}{2 b}\right\}\right] .
\end{aligned}
$$

Rewriting Eqn. (25) results in

$$
\begin{aligned}
& L_{(a, b, c, d)}(h(x)) \\
& =\frac{\sqrt{j 2 \pi b}}{j 2 \pi b} \int_{-\infty}^{\infty} \int_{-\infty}^{\infty} \mathrm{d} x \mathrm{~d} y\langle p|K| x\rangle\langle x \mid g\rangle \\
& \quad \cdot\langle p|K| y\rangle\langle y \mid f\rangle \exp \left[\frac{-j d p^{2}}{2 b}\right] .
\end{aligned}
$$


Rearranging Eqn. (26), we get

$$
\begin{aligned}
& L_{(a, b, c, d)}(h(x)) \\
& =\sqrt{j 2 \pi b}\left[\frac{1}{\sqrt{j 2 \pi b}} \int_{-\infty}^{\infty} \mathrm{d} x\langle p|K| x\rangle\langle x \mid g\rangle\right. \\
& \left.\cdot \frac{1}{\sqrt{j 2 \pi b}} \int_{-\infty}^{\infty} \mathrm{d} y\langle p|K| y\rangle\langle y \mid f\rangle \cdot \exp \left\{\frac{-j d p^{2}}{2 b}\right\}\right] .
\end{aligned}
$$

Rewriting Eqn. (27) results in

$$
\begin{aligned}
& L_{(a, b, c, d)}(h(x)) \\
& =\sqrt{j 2 \pi b} e^{-\frac{j}{2}\left(\frac{d}{b} p^{2}\right)} F_{(a, b, c, d)}(p) G_{(a, b, c, d)}(p) .
\end{aligned}
$$

This is just a new convolution theorem under the LCT, derived by representation transformation in quantum mechanics. Hence Eqn. (15) is obtained and the theorem is proved.

The reciprocal transform of Eqn. (28) can be obtained by writing the definition of the inverse LCT and is given by

$$
L_{(a, b, c, d)}^{-1}(H(p))=\int_{-\infty}^{\infty} \mathrm{d} p\langle p|K| x\rangle^{*} \cdot H_{(a, b, c, d)}(p),
$$

where $*$ indicates the complex conjugate. By using the theory of representation transformation in quantum mechanics, Eqn. (29) results in

$$
\begin{aligned}
L^{-1} & \\
& =\int_{-\infty}^{\infty} \mathrm{d} p\left\langle x\left|K^{\dagger}\right| p\right\rangle \cdot\langle p \mid H\rangle \\
& =\int_{-\infty}^{\infty} \mathrm{d} p\left\langle x\left|K^{\dagger} K\right| h\right\rangle=\langle x \mid h\rangle=h(x) .
\end{aligned}
$$

Seeing FT as a special case of the LCT, when $(a, b, c, d)=(0,1,-1,0)$, Eqn. (28) becomes

$L_{(0,1,-1,0)}(h(x))=\sqrt{j 2 \pi} F_{(0,1,-1,0)}(p) G_{(0,1,-1,0)}(p)$.

Similarly, seeing the FRFT as a special case of the LCT, when $(a, b, c, d)=(\cos \alpha, \sin \alpha,-\sin \alpha, \cos \alpha)$, Eqn. (28) becomes

$$
F_{\alpha}(h(x))=\sqrt{j 2 \pi \sin \alpha} e^{-\frac{j}{2} p^{2} \cot \alpha} F_{\alpha}(p) G_{\alpha}(p),
$$

and the FT as a special case of the FRFT, $\alpha=\pi / 2$, (32) becomes

$$
F_{\pi / 2}(h(x))=\sqrt{j 2 \pi} F_{\pi / 2}(p) G_{\pi / 2}(p) .
$$

Equations (31) and (32) are special cases of the LCT.

\section{Proposed transform of a product by coordinate-momentum representation transform}

The one-dimensional linear canonical transform of $m(x)$ in the context of quantum mechanics is given by

$$
L_{(a, b, c, d)}(m(x))=\frac{1}{\sqrt{j 2 \pi b}} \int_{-\infty}^{\infty} \mathrm{d} x\langle p|K| x\rangle\langle x \mid m\rangle .
$$

From the definition of the modified product theorem as given by Eqn. (16),

$$
\begin{aligned}
& L_{(a, b, c, d)}(m(x)) \\
& =\frac{1}{\sqrt{j 2 \pi b}} \int_{-\infty}^{\infty} \mathrm{d} x\langle x \mid f\rangle\langle x \mid g\rangle\langle p|K| x\rangle \\
& \quad \cdot \exp \left[j \frac{a}{2 b} x^{2}\right],
\end{aligned}
$$

the one-dimensional inverse linear canonical transform in the context of quantum mechanics is given by

$$
\langle x \mid g\rangle=\sqrt{\frac{-1}{j 2 \pi b}} \int_{-\infty}^{\infty} \mathrm{d} v\langle v \mid G\rangle\langle v|K| x\rangle^{*}
$$

where * indicates the complex conjugate. Rewriting Eqn. (36) results in

$$
\langle x \mid g\rangle=\sqrt{\frac{-1}{j 2 \pi b}} \int_{-\infty}^{\infty} \mathrm{d} v\langle v \mid G\rangle\left\langle x\left|K^{\dagger}\right| v\right\rangle .
$$

Substituting the value of Eqn. (37) in (35) results in

$$
\begin{aligned}
& L_{(a, b, c, d)}(m(x)) \\
& =\left|\frac{1}{2 \pi b}\right| \int_{-\infty}^{\infty} \int_{-\infty}^{\infty} \mathrm{d} v \mathrm{~d} x\langle v \mid G\rangle \\
& \quad \cdot\left\langle x\left|K^{\dagger}\right| v\right\rangle\langle x \mid f\rangle \cdot\langle p|K| x\rangle \exp \left[j \frac{a}{2 b} x^{2}\right] .
\end{aligned}
$$

Solving for $\left\langle x\left|K^{\dagger}\right| v\right\rangle \cdot\langle p|K| x\rangle$ results in

$$
\begin{gathered}
\Rightarrow \exp \left[\frac{-j d v^{2}+2 j v x-j a x^{2}}{2 b}\right] \\
\cdot \exp \left[\frac{j d p^{2}-2 j p x+j a x^{2}}{2 b}\right] .
\end{gathered}
$$


Substituting the value of Eqn. (39) in (38) results in

$$
\begin{aligned}
& L_{(a, b, c, d)}(m(x)) \\
& =\left|\frac{1}{2 \pi b}\right| \int_{-\infty}^{\infty} \int_{-\infty}^{\infty} \mathrm{d} v \mathrm{~d} x\langle v \mid G\rangle\langle x \mid f\rangle \cdot \exp \left[j \frac{a}{2 b} x^{2}\right] \\
& \cdot \exp \left[\frac{-j d v^{2}+2 j v x-j a x^{2}}{2 b}\right] \\
& \cdot \exp \left[\frac{j d p^{2}-2 j p x+j a x^{2}}{2 b}\right] .
\end{aligned}
$$

Multiplying and dividing Eqn. (40) by $\exp \left[\frac{-j}{2} \frac{d}{b} v(v-2 p)\right]$ results in

$$
\begin{aligned}
& L_{(a, b, c, d)}(m(x)) \\
& =\left|\frac{1}{2 \pi b}\right| \int_{-\infty}^{\infty} \int_{-\infty}^{\infty} \mathrm{d} v \mathrm{~d} x\langle v \mid G\rangle\langle x \mid f\rangle \\
& \quad \cdot \exp \left[\frac { - j } { 2 } \left\{\frac{d v^{2}-2 v x-d p^{2}+2 p x-a x^{2}}{b}\right.\right. \\
& \left.\left.+\frac{d v^{2}-2 d p v-d v^{2}+2 d p v}{b}\right\}\right] .
\end{aligned}
$$

Rewriting Eqn. (41), we get

$$
\begin{aligned}
& L_{(a, b, c, d)}(m(x)) \\
& =\left|\frac{1}{2 \pi b}\right| \int_{-\infty}^{\infty} \int_{-\infty}^{\infty} \mathrm{d} v \mathrm{~d} x\langle v \mid G\rangle\langle x \mid f\rangle \\
& \quad \cdot \exp \left[\frac{j}{2}\left\{\frac{d}{b}\left(p^{2}+v^{2}-2 p v\right)-\frac{2 x}{b}(p-v)+\frac{a x^{2}}{b}\right\}\right] \\
& \cdot \exp \left[-j \frac{d}{b} v^{2}+j \frac{d}{b} p v\right] .
\end{aligned}
$$

Rewriting Eqn. (42) results in

$$
\begin{aligned}
& L_{(a, b, c, d)}(m(x)) \\
& =\left|\frac{1}{2 \pi b}\right| \int_{-\infty}^{\infty} \int_{-\infty}^{\infty} \mathrm{d} v \mathrm{~d} x\langle v \mid G\rangle\langle x \mid f\rangle \\
& \cdot \exp \left[\frac{j}{2}\left\{\frac{d}{b}(p-v)^{2}-\frac{2 x}{b}(p-v)+\frac{a x^{2}}{b}\right\}\right] . \\
& \cdot \exp \left[j \frac{d}{b} v(p-v)\right] .
\end{aligned}
$$

From the representation theory of quantum mechanics,
Eqn. (43) can be written as

$$
\begin{aligned}
& L_{(a, b, c, d)}(m(x)) \\
& =\sqrt{\frac{-1}{j 2 \pi b}} \int_{-\infty}^{\infty} \mathrm{d} v\langle v \mid G\rangle\langle p-v \mid f\rangle \\
& \cdot \exp \left[j \frac{d}{b} v(p-v)\right] \\
& =G_{(a, b, c, d)}(p) \otimes F_{(a, b, c, d)}(p) \\
& \cdot \exp \left[j \frac{d}{b} v p\right] .
\end{aligned}
$$

This is just a new product theorem under the LCT, derived by representation in quantum mechanics. Hence Eqn. (16) is solved and the theorem is proved.

\section{Simulation results}

The convolution theorem in the LCT domain given by Deng et al. (2006) and Wei et al. (2012; 2009) is compared with the proposed convolution theorem by simulating on system with an Intel core $\mathrm{cm}^{\mathrm{TM}} \mathrm{i3}-330 \mathrm{M}$ $2.13 \mathrm{GHz}$ processor with $3 \mathrm{~GB}$ RAM. The convolution operation of a rectangular function $x(t)$ of unit amplitude is performed with itself, i.e., $(x \otimes x)(t)$. As a result of the convolution operation, one obtains a triangular (Bartlett) function with a duration double of that of the rectangular function as shown in Fig. 1. Then the LCT of convolution operations defined by Deng et al. (2006) and Wei et al. (2012; 2009) is compared with the LCT of the proposed convolution operation for $(a, b, c, d)=(0.707,0.707,-0.707,0.707)$ and $(a, b, c, d)=(0.5,0.866,-0.866,0.5)$ as shown in Figs. 2 and 3 , respectively. Simultaneously, the LCT of the triangular function is also evaluated for the same values of $(a, b, c, d)$ to make a comparison. It is shown that the real (Re), imaginary ( $\mathrm{Im}$ ) and absolute (Abs) components of the proposed convolution theorem for the LCT resemble maximally the different components of the LCT of a triangular function for $(a, b, c, d)=(0.707,0.707,-0.707,0.707)$ and $(a, b, c, d)=(0.5,0.866,-0.866,0.5)$.

\section{Application}

To illustrate the proposed work, an example of filtering is presented. For filtering applications, the time-frequency plot of the original signal and the corrupted signal plays an important role. Many time-frequency signal representations are available, such as the Wigner Distribution (WD) (Wigner, 1932; Hlawatsch and Boudreaux-Bartels, 1992; Classen and Mecklenbrauker, 1980), the Short Time Fourier Transform (STFT) (Hlawatsch and Boudreaux-Bartels, 1992; Portnoff, 1980; Cohen, 1989), the ambiguity function (Hlawatsch 


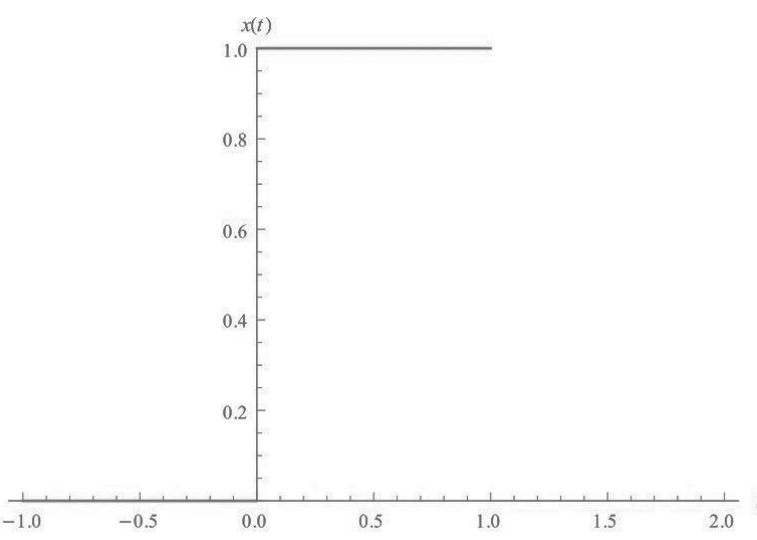

(a)

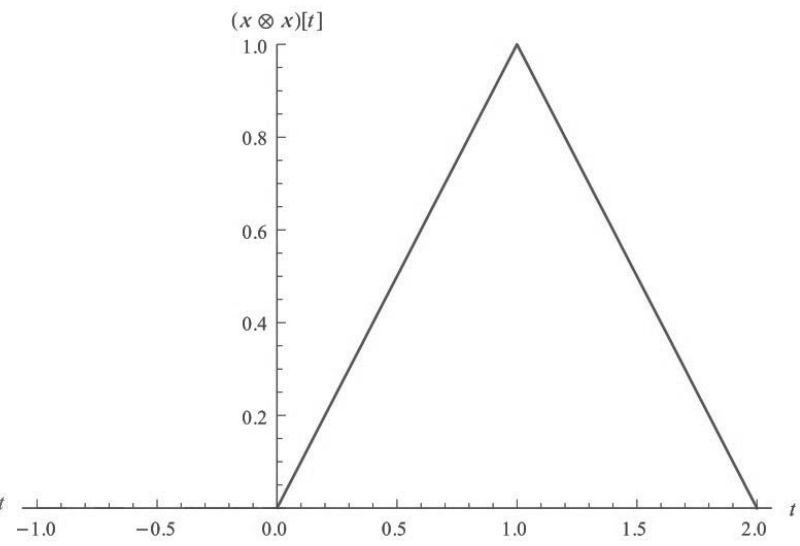

(b)

Fig. 1. Rectangular function $x(t)$ (a), convolved signal $(x \otimes x)(t)$, i.e., triangular (Bartlett) window (b).

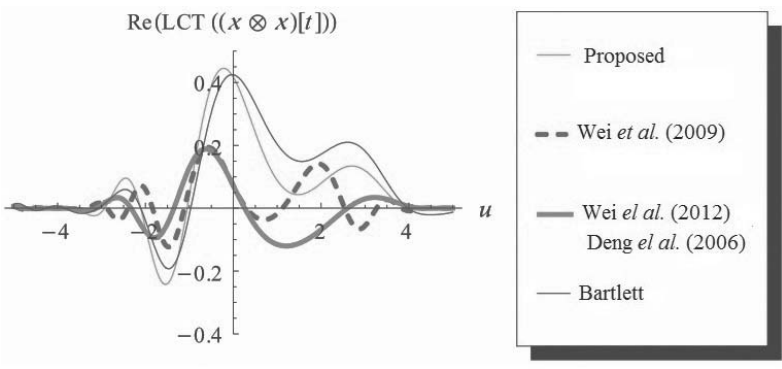

(a)

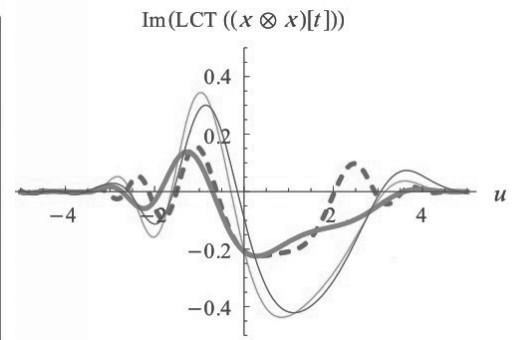

(b)
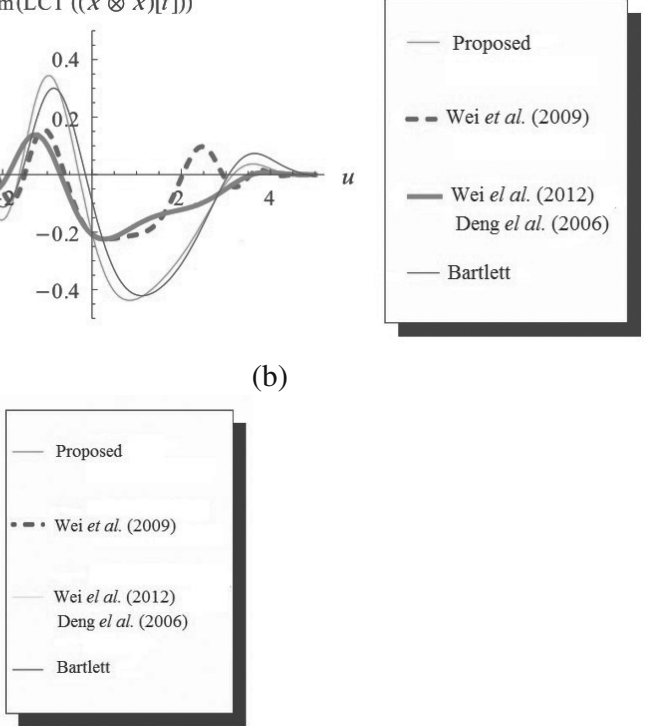

(c)

Fig. 2. Real value (a), imaginary value (b), absolute value of the LCT of $(x \otimes x)(t)$ by using the Deng et al. (2006) and Wei et al. (2012; 2009), Bartlett and proposed methods for $(a, b, c, d)=(0.707,0.707,-0.707,0.707)$ (c).

and Boudreaux-Bartels, 1992; Cohen, 1989), the Gabor transform (Swiercz, 2010) and the spectrogram (Hlawatsch and Boudreaux-Bartels, 1992; Portnoff, 1980; Cohen, 1989). Out of these techniques, the WD is a powerful (Bouachache and Rodriguez, 1984) time-frequency analysis tool and is used in this paper. The concept of the canonical filter design is understood from the definition of canonical convolution (Pei and Deng, 2001), given by

$$
\begin{aligned}
& g(t) \\
& =L_{(d,-b,-c, a)}\left(L_{(a, b, c, d)}(h(t)) \cdot L_{(a, b, c, d)}(x(t))\right),
\end{aligned}
$$

where

$$
H(u)=L_{(a, b, c, d)}(h(t))
$$

represents the transfer function of the pass-stop band canonical filter and

$$
L_{(d,-b,-c, a)}\left(L_{(a, b, c, d)}(f(t))\right)=f(t)
$$

gives the reversibility property of the LCT. Consider an original signal

$$
x(t)=2 \exp \left[-\frac{1}{8}(t+1)^{2}-j 0.5 t^{2}\right] .
$$

Let this signal be corrupted by an Additive White Gaussian Noise (AWGN) of $5 \mathrm{~dB}$ Signal to Noise Ratio 


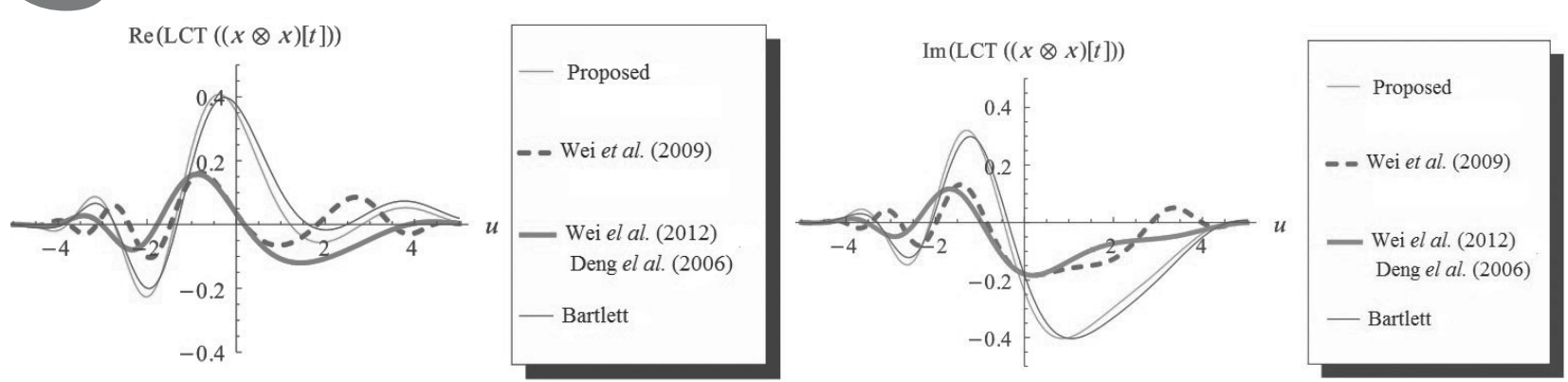

(a)

(b)
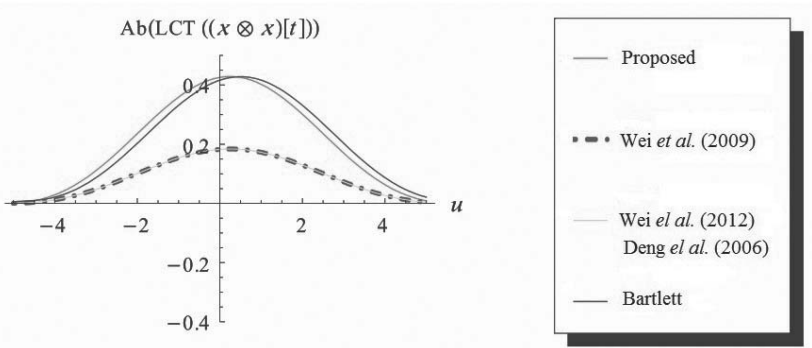

(c)

Fig. 3. Real value (a), imaginary value (b), absolute value of the LCT of $(x \otimes x)(t)$ by using the Deng et al. (2006) and Wei et al. (2012; 2009), Bartlett and proposed methods for $(a, b, c, d)=(0.5,0.866,-0.866,0.5)$ (c).

(SNR). As shown in Fig. 4(a), the original signal $x(t)$ is plotted. Figure 4(c) shows the corrupted signal. Figures 4(b) and 4(d) show the WD of the original signal and corrupted signal, respectively. Following the method given by Pei and Deng (2001), we found the optimal filtering domain is the canonical domain with $(a, b, c, d)=(0.16,1,-1,0)$. Figures 4(e) and 4(f) show a comparison between the imaginary and real part of the exact signal and the proposed signal output of the filter with the Mean Square Error (MSE) equal to 0.00049 . As a comparison with the convolution theorems already derived, the number of chirp functions required by the proposed convolution process is two (cf. Eqn. (18)) and seven (cf. Eqn. (24)), compared with seven and six chirp functions by Wei et al. (2009) or three and seven chirp functions by Deng et al. (2006) for the left and right-hand sides of the convolution process, respectively. Hence the proposed convolution theorem also reduces the computational complexity.

\section{Conclusion}

We defined the convolution and product operations of two functions in the LCT domain and derived the convolution and product theorem for linear canonical transformation by employing the coordinate-momentum representation and the LCT operator. The derivation is direct and concise since we used Dirac's representation theory (Huang et al., 2006). It is seen from the results of Figs. 2 and 3 that the proposed weighted convolution theorem gives better results than the convolution theorem by Deng et al. (2006) and Wei et al. (2012; 2009), as the results given by the proposed theorem resemble maximally the shape of the LCT of the triangular function. Further, an application of filtering was presented with the proposed convolution theorem and it was found that with the help of proposed theorem the signal is recovered with a minimum mean square error. The derived approach can be used for defining the canonical correlation theorem for the LCT, which will play an important role in signal spectrum analysis, beamforming and signal detection in the canonical domain.

\section{Acknowledgment}

The authors thankfully acknowledge the reviewers and the editor-in-chief for their valuable comments and suggestions in shaping this paper into its present form.

\section{References}

Abe, S. and Sheridan, J. (1994a). Generalization of the fractional Fourier transformation to an arbitrary linear lossless transformation: An operator approach, Journal of Physics, A: Mathematical and General 27(12): 4179-4187.

Abe, S. and Sheridan, J. (1994b). Optical operations on wave functions as the Abelian subgroups of the special affine Fourier transformation, Optics Letters 19(22): 1801-1803.

Alieva, T. and Bastiaans, M. (1999). Powers of transfer matrices determined by means of eigenfunctions, Journal of Optical Society of America A 16(10): 2413-2418. 


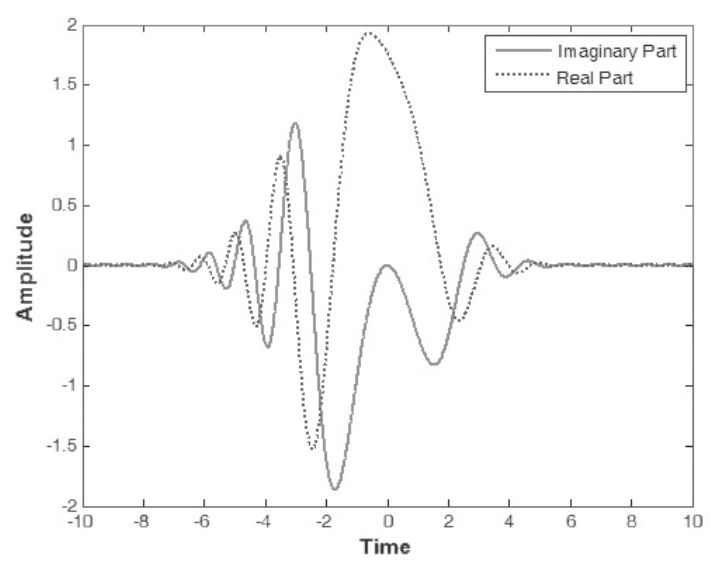

(a)

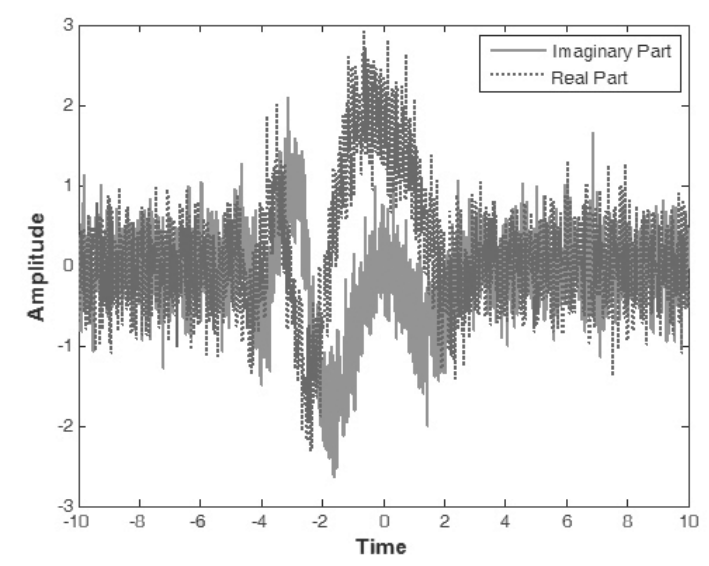

(c)

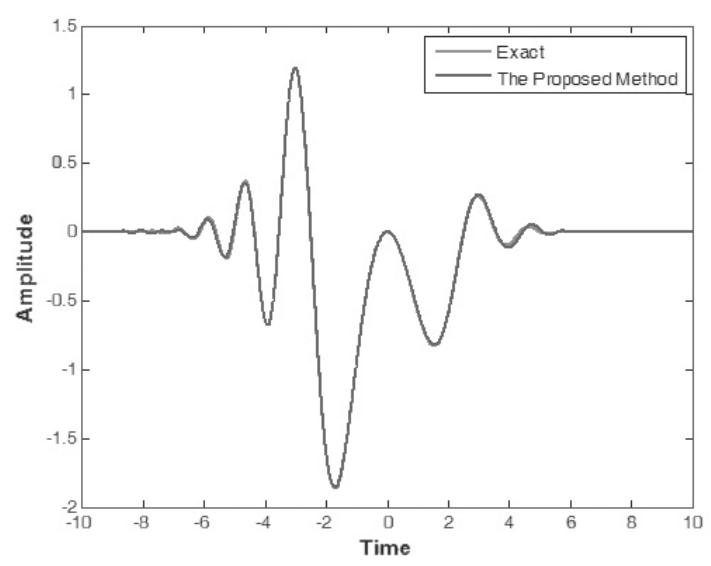

(e)

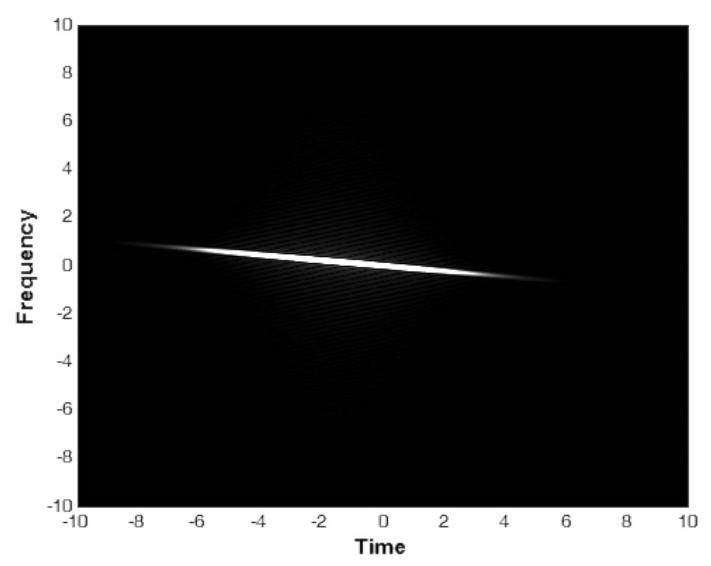

(b)

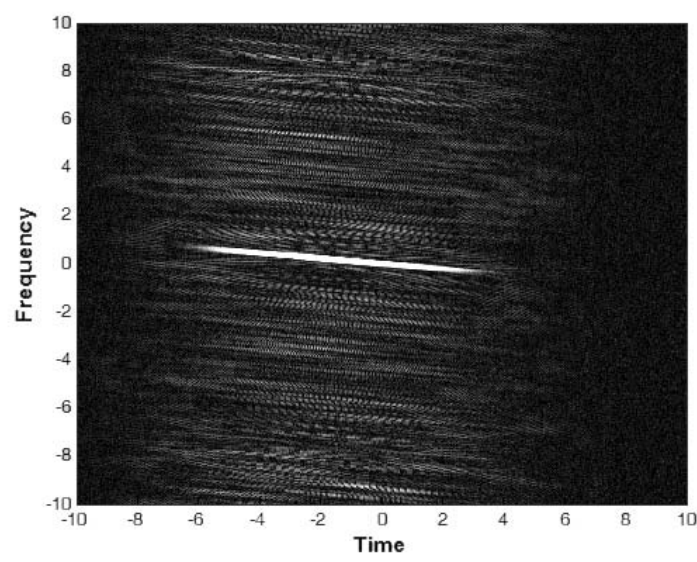

(d)

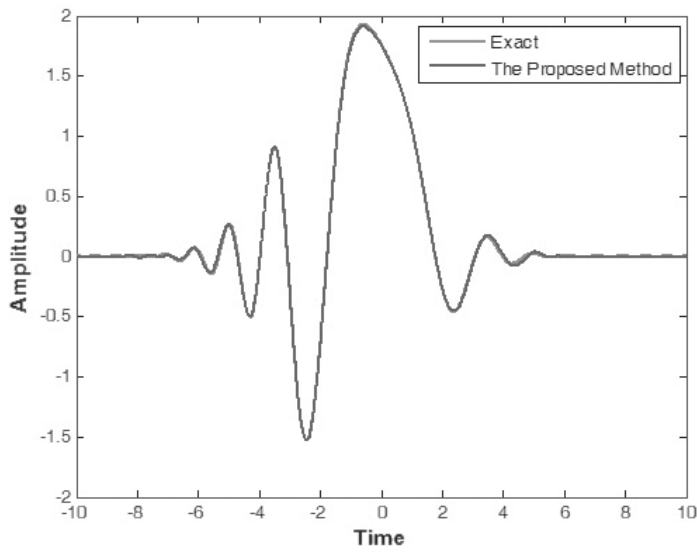

(f)

Fig. 4. Original signal (a), WD of the original signal (b), corrupted signal (c), WD of the corrupted signal (d), imaginary part: comparison of the recovered and original signals (e), real part: comparison of the recovered and original signals (f).

Almeida, L. (1994). The fractional Fourier transform and time-frequency representations, IEEE Transactions on Signal Processing 42(11): 3084-3091.

Almeida, L. (1997). Product and convolution theorems for the fractional Fourier transform, IEEE Signal Processing Letters 4(1): 15-17.

Barshan, B., Ozaktas, H. and Kutey, M. (1997). Optimal filters with linear canonical transformations, Optics Communica- 
tions 135(1-3): 32-36.

Bastiaans, M. (1979). Wigner distribution function and its application to first-order optics, Journal of Optical Society of America 69(12): 1710-1716.

Bernardo, L. (1996). ABCD matrix formalism of fractional Fourier optics, Optical Engineering 35(03): 732-740.

Bouachache, B. and Rodriguez, F. (1984). Recognition of time-varying signals in the time-frequency domain by means of the Wigner distribution, IEEE International Conference on Acoustics, Speech, and Signal Processing, San Diego, CA, USA, Vol. 9, pp. 239-242.

Classen, T.A.C.M. and Mecklenbrauker, W.F.G. (1980). The Wigner distribution: A tool for time-frequency signal analysis, Part I: Continuous time signals, Philips Journal of Research 35(3): 217-250.

Cohen, L. (1989). Time-frequency distributions: A review, Proceedings of the IEEE 77(7): 941-981.

Collins, S. (1970). Lens-system diffraction integral written in terms of matrix optics, Journal of Optical Society of America 60(9): 1168-1177.

Deng, B., Tao, R. and Wang, Y. (2006). Convolution theorem for the linear canonical transform and their applications, Science in China, Series F: Information Sciences 49(5): 592-603.

Gdawiec, K. and Domańska, D. (2011). Partitioned iterated function systems with division and a fractal dependence graph in recognition of 2D shapes, International Journal of Applied Mathematics and Computer Science 21(4): 757-767, DOI: 10.2478/v10006-011-0060-8.

Goel, N. and Singh, K. (2011). Analysis of Dirichlet, generalized Hamming and triangular window functions in the linear canonical transform domain, Signal, Image and Video Processing DOI: 10.1007/s11760-011-0280-2.

Healy, J. and Sheridan, J. (2008). Cases where the linear canonical transform of a signal has compact support or is band-limited, Optics Letters 33(3): 228-230.

Healy, J. and Sheridan, J.T. (2009). Sampling and discretization of the linear canonical transform, Signal Processing 89(4): 641-648.

Hennelly, B. and Sheridan, J.T. (2005a). Fast numerical algorithm for the linear canonical transform, Journal of Optical Society of America A 22(5): 928-937.

Hennelly, B. and Sheridan, J.T. (2005b). Generalizing, optimizing, and inventing numerical algorithms for the fractional Fourier, Fresnel, and linear canonical transforms, Journal of Optical Society of America A 22(5): 917-927.

Hlawatsch, F. and Boudreaux-Bartels, G.F. (1992). Linear and quadratic time-frequency signal representation, IEEE Signal Processing Magazine 9(2): 21-67.

Hong-yi, F., Ren, H. and Hai-Liang, L. (2008). Convolution theorem of fractional Fourier transformation derived by representation transformation in quantum mechanics, Communication Theoretical Physics 50(3): 611-614.
Hong-yi, F. and VanderLinde, J. (1989). Mapping of classical canonical transformations to quantum unitary operators, Physical Review A 39(6): 2987-2993.

Hong-yi, F. and Yue, F. (2003). New eigenmodes of propagation in quadratic graded index media and complex fractional Fourier transform, Communication Theoretical Physics 39(1): 97-100.

Hong-yi, F. and Zaidi, H. (1987). New approach for calculating the normally ordered form of squeeze operators, Physical Review D 35(6): 1831-1834.

Hua, J., Liu, L. and Li, G. (1997). Extended fractional Fourier transforms, Journal of Optical Society of America A 14(12): 3316-3322.

Huang, J. and Pandić, P. (2006). Dirac Operators in Representation Theory, Birkhauser/Springer, Boston, MA/ New York, NY.

James, D. and Agarwal, G. (1996). The generalized Fresnel transform and its applications to optics, Optics Communications 126(4-6): 207-212.

Kiusalaas, J. (2010). Numerical Methods in Engineering with Python, Cambridge University Press, New York, NY.

Koc, A., Ozaktas, H., Candan, C. and Kutey, M. (2008). Digital computation of linear canonical transforms, IEEE Transactions on Signal Processing 56(6): 2383-2394.

Li, B., Tao, R. and Wang, Y. (2007). New sampling formulae related to linear canonical transform, Signal Processing 87(5): 983-990.

Moshinsky, M. and Quesne, C. (1971). Linear canonical transformations and their unitary representations, Journal of Mathematical Physics 12(8): 1772-1783.

Namias, V. (1979). The fractional order Fourier transform and its application to quantum mechanics, IMA Journal of Applied Mathematics 25(3): 241-265.

Nazarathy, M. and Shamir, J. (1982). First-order optics-a canonical operator representation: Lossless systems, Journal of Optical Society of America A 72(3): 356-364.

Ogura, A. (2009). Classical and quantum ABCD-transformation and the propagation of coherent and Gaussian beams, Journal of Physics, B: Atomic, Molecular and Optical Physics 42(14): 145504, DOI:10.1088/0953-4075/42/14/145504.

Ogura, A. and Sekiguchi, M. (2007). Algebraic structure of the Feynman propagator and a new correspondence for canonical transformations, Journal of Mathematical Physics 48(7): 072102, DOI:10.1063/1.2748378.

Oktem, F. and Ozaktas, H. (2010). Equivalence of linear canonical transform domains to fractional Fourier domains and the bicanonical width product: A generalization of the space-bandwidth product, Journal of Optical Society of America A 27(8): 1885-1895.

Ozaktas, H., Barshan, B., Mendlovic, D. and Onural, L. (1994). Convolution, filtering, and multiplexing in fractional Fourier domains and their relationship to chirp and wavelet transforms, Journal of Optical Society of America A 11(2): 547-559. 
Ozaktas, H., Kutey, M. and Zalevsky, Z. (2000). The Fractional Fourier Transform with Applications in Optics and Signal Processing, John Wiley and Sons, New York, NY.

Palma, C. and Bagini, V. (1997). Extension of the Fresnel transform to ABCD systems, Journal of Optical Society of America A 14(8): 1774-1779.

Pei, S. and Ding, J.J. (2001). Relations between fractional operations and time-frequency distributions, and their applications, IEEE Transactions on Signal Processing 49(8): 1638-1655.

Pei, S. and Ding, J.J. (2002a). Closed-form discrete fractional and affine Fourier transforms, IEEE Transactions on Signal Processing 48(5): 1338-1353.

Pei, S. and Ding, J.J. (2002b). Eigenfunctions of linear canonical transform, IEEE Transactions on Signal Processing 50(1): 11-26.

Portnoff, M. (1980). Time-frequency representation of digital signals and systems based on short-time Fourier analysis, IEEE Transactions on Acoustics, Speech and Signal Processing 28(1): 55-69.

Puri, R. (2001). Mathematical Methods of Quantum Optics, Springer-Verlag, Berlin/Heidelberg.

Sharma, K. and Joshi, S. (2006). Signal separation using linear canonical and fractional Fourier transforms, Optics Communications 265(2): 454-460.

Sharma, K. and Joshi, S. (2007). Papoulis-like generalized sampling expansions in fractional Fourier domains and their application to super resolution, Optics Communications 278(1): 52-59.

Singh, A. and Saxena, R. (2012). On convolution and product theorems for FRFT, Wireless Personal Communications 65(1): 189-201.

Stern, A. (2006). Sampling of linear canonical transformed signals, Signal Processing 86(7): 1421-1425.

Świercz, E. (2010). Classification in the Gabor time-frequency domain of non-stationary signals embedded in heavy noise with unknown statistical distribution, International Journal of Applied Mathematics and Computer Science 20(1): 135-147, DOI: 10.2478/v10006-010-0010-x.

Shin, Y.J. and Park, C.H. (2011). Analysis of correlation based dimension reduction methods, International Journal of Applied Mathematics and Computer Science 21(3): 549-558, DOI: 10.2478/v10006-011-0043-9.

Tao, R., Li, B., Wang, Y. and Aggrey, G. (2008). On sampling of bandlimited signals associated with the linear canonical transform, IEEE Transactions on Signal Processing 56(11): 5454-5464.

Tao, R., Qi, L. and Wang, Y. (2004). Theory and Applications of the Fractional Fourier Transform, Tsinghua University Press, Beijing.
Wei, D., Ran, Q. and Li, Y. (2012). A convolution and correlation theorem for the linear canonical transform and its application, Circuits, Systems, and Signal Processing 31(1): 301-312.

Wei, D., Ran, Q., Li, Y., Ma, J. and Tan, L. (2009). A convolution and product theorem for the linear canonical transform, IEEE Signal Processing Letters 16(10): 853-856.

Wigner, E. (1932). On the quantum correlation for thermodynamic equilibrium, Physical Review 40(5): 749-759.

Wolf, K. (1979). Integral Transforms in Science and Engineering, Plenum Press, New York, NY.

Zayad, A. (1998). A product and convolution theorems for the fractional Fourier transform, IEEE Signal Processing Letters 5(4): 101-103.

Zhang, W., Feng, D. and Gilmore, R. (1990). Coherent states: Theory and some applications, Reviews of Modern Physics 62(4): 867-927.

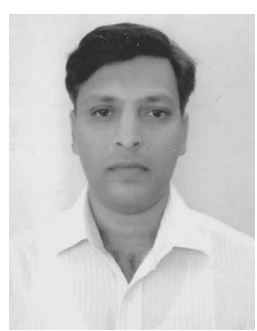

Navdeep Goel completed his B.E. in electronics and telecommunication engineering in 2002 at BNCOE, Pusad (Amravati University), Maharastra, India, his M.Tech. in electronics and communication engineering at Punjab Technical University, Jalandhar (Punjab), and is pursuing a Ph.D. at Thapar University, Patiala (Punjab). He has worked as a scientist/engineer (SC) in the Vikram Sarabhai Space Centre, Indian Space Research Organization (ISRO), Thiruvananthapuram. He has been teaching since 2004 and is presently working as an assistant professor (ECE) in the Yadavindra College of Engineering, Punjabi University Guru Kashi Campus, Talwandi Sabo, Bathinda, Punjab.

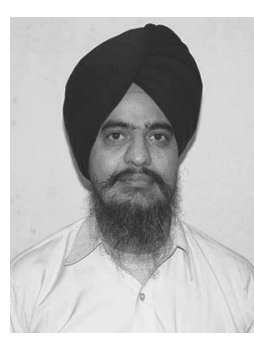

Kulbir Singh was born in Batala, Punjab, India. He completed his B.Tech. degree in 1997 at PTU, Jalandhar. He received his M.E. and Ph.D. degrees from Thapar University, Patiala, in 2000 and 2006, respectively. He is currently working as an associate professor in the Department of Electronics and Communication Engineering, Thapar University, Patiala. He has published more than 55 research papers in national and international journals/conference proceedings. He is a recipient of the Best Paper Award of the IETE Journal of Education for the year 2008. His research interests include signal processing, image processing, DSP processors based design and fractional transforms.

Received: 11 July 2012

Revised: 22 November 2012 